\title{
Molecular survey and microscopic examination of Hepatozoon Miller, 1908 (Apicomplexa: Adeleorina) in lacertid lizards from the western Mediterranean
}

\author{
João P.M.C. Maia ${ }^{1,2}$, Ana Perera ${ }^{1}$ and D. James Harris ${ }^{1}$ \\ ${ }^{1}$ CIBIO-UP, Centro de Investigação em Biodiversidade e Recursos Genéticos da Universidade do Porto, Campus Agrário de \\ Vairão, 4485-661 Vairão, Portugal; \\ ${ }^{2}$ Departamento de Biologia, Faculdade de Ciências, Universidade do Porto, Rua do Campo Alegre FC4, 4169-007 Porto, Portugal
}

\begin{abstract}
The genus Hepatozoon Miller, 1908 (Apicomplexa: Adeleorina) is composed of intracellular haemogregarine parasites that are widely distributed among all tetrapod groups. The present study combines microscopic and molecular data on haemogregarine parasites from lizards in the western Mediterranean. We screened tissue samples and examined blood smears for the presence of species of Hepatozoon from four lizards, namely Algyroides marchi Valverde, endemic to Southeast Spain, Podarcis bocagei Seoane from Spain and Portugal, P. hispanica Steindachner from Spain, and P. lilfordi Günther from Cabrera, Balearic Islands (Spain). Our results show that prevalence and intensity of Hepatozoon parasites vary between and within lizard species from different regions. Algyroides marchi and P. bocagei from Spain had the lowest values, whereas $P$. hispanica had the highest. Phylogeny based on 18S rRNA gene sequences indicates that most of the new Hepatozoon sequences are part of a clade exclusive from North African and Iberian lizards, except for a single P. bocagei isolate that is found related to another clade including isolates from other reptile host species and rodents. Interestingly, isolates from Algyroides form a distinct monophyletic subgroup, which could be a signal of strict host-specificity within this host genus.
\end{abstract}

Keywords: Apicomplexa, haemogregarine, 18S rRNA, phylogeny, tissue, blood smears

The study of parasites is important not only in terms of understanding biodiversity as a whole but also for seeking answers to more complex questions related to hostspecificity and co-evolution (Poulin and Mouillot 2005, Paterson and Piertney 2011). However, there is a bias within the study of parasites, with most research focusing on parasites that are considered of great veterinary, medical and public health importance. Hence, most information available is dedicated to parasites affecting domestic animals rather than wild species, with parasites infecting groups such as reptiles being still poorly studied.

Haemogregarines represent an important parasite group with veterinary importance for some groups of their hosts, such as dogs (Baneth et al. 2003), and they are one of the most common parasites found in reptiles. Haemogregarines are a group of apicomplexan (Apicomplexa, Adeleorina) intracellular parasites and four genera within this group are known to infect reptiles: Hepatozoon Miller, 1908, Haemogregarina Danilewsky, 1885, Karyolysus Labbé, 1894 and Hemolivia Petit, Landau, Baccam et Lainson, 1990 (Smith 1996, Smith and Desser 1997, Telford 2009). The genus Hepatozoon is the most widely distributed among reptiles and also has been reported in all other tetrapod groups (Telford 2009). Lizards of sev- eral genera, e.g., Iberolacerta Arribas, Podarcis Wagler, Psammodromus Fitzinger and Timon Tschudi, from the Iberian Peninsula have been shown to have high levels of haemogregarine infection using traditional blood smear inspection methods (Álvarez-Calvo 1975, Amo et al. 2004, 2005a, b, Jovani et al. 2004, Martín et al. 2007, Roca and Galdón 2010, Sacchi et al. 2011). This makes lizards from this region potentially good models for studying host-parasite interactions. In contrast, to our knowledge there are no studies on the prevalence and intensity of Hepatozoon in species of Algyroides Bibron et Bory de Saint-Vicent from the Iberian Peninsula.

Molecular data of Hepatozoon parasites in public databases, such as GenBank, are also mainly biased towards socio-economically important species, especially domestic and wild mammals. This is also the case of the Iberian Peninsula, for which multiple Hepatozoon parasites have been found in mammals and their definitive hosts, i.e. arthropods (Garcia et al. 1990, Criado-Fornelio et al. 2003, 2006, 2007, 2009, Ortuno et al. 2008, Tabar et al. 2008, Yabsley et al. 2008, Lledó et al. 2010). Although Hepatozoon sequences from reptiles are scarce in GenBank, there are a few from snakes (Ujvari et al. 2004, Harris et al. 2011, Moço et al. 2012, Tomé et al. 2012) and liz- 
ards, with records from the Seychelles (Harris et al. 2011), North Africa (Maia et al. 2011) and the Iberian Peninsula and Balearic Islands (Harris et al. 2012). These studies have shown that $18 \mathrm{~S}$ rRNA gene primers successfully amplify Hepatozoon from tail tips collected from lizards, highlighting the utility of this method for screening and providing new insights into parasite phylogeny.

Hepatozoon currently includes more than 300 species (Smith 1996) with variable morphological characteristics, diverse life histories (Smith and Desser 1997) and a wide range of host species. Nevertheless, many of these species have been described solely on morphological characteristics of the parasites found in different host species, which may be problematic if host specificity is low (Mathew et al. 2000). Based on diverse morphological and developmental characteristics, Smith and Desser (1997) suggested that the genus should be partitioned into two genera of adeleorin parasites, which was supported by a recent study on the phylogeny of the adeleorinid coccidia, which has indicated paraphyly of Hepatozoon (Barta et al. 2012).

In addition, Barta et al. (2012) showed that these parasites seem to have a relatively high level of host-parasite specificity with their definitive hosts (invertebrates) rather than intermediate hosts (vertebrates), in particular with the separation of parasites from leeches as a distinct clade from those from arthropods. Although four main lineages based on 18S rRNA gene sequences have been described in lizards so far (Maia et al. 2011), the phylogenetic relationships within Hepatozoon seem to be complex and remain largely unresolved.

The aim of this study is to increase the knowledge of haemogregarines in lizards from the Iberian Peninsula and Cabrera Island by combining both molecular and morphological methods, and to relate this information with the current literature on Hepatozoon. When possible, parasite load was quantified from positive samples.

\section{MATERIALS AND METHODS}

\section{Sample collection}

Blood smears and tissue samples were collected from lizards from different localities from the Iberian Peninsula: Gião and Viana do Castelo (Portugal), Alba de Tormes, Tanes, Palacios de Compludo, Albacete, Jaén, Rambla los Vaquerizos and Pedro Andrés (all from Spain) and Cabrera (Balearic Islands, Spain). Species were identified by experienced herpetologists in the field, body size (snout-vent length, SVL, to the nearest millimetre) and sex (based on colour pattern and the existence of developed femoral pores) of the host were also registered. After sample collection, animals were released at the capture site.

Tissue samples were preserved for molecular analysis (tail tips containing blood stored in $96 \%$ ethanol) and used to screen the presence of Hepatozoon parasites. When enough blood was available from the autotomized tail, blood smears were made, then air-dried, fixed with methanol and stained with Giemsa (Telford 2009). A total of 104 tissue samples from two species were collected (Table 1). Additionally, 19 Podarcis hispanica Steindachner type 1b samples from Spain (June 2009) and ten
P. lilfordi Günther samples from Cabrera Island (September 2010) included in Harris et al. (2012) were also used in this study for microscopic examination and further molecular and phylogenetic analyses.

\section{DNA extraction, amplification and sequencing}

DNA was extracted from tissue using standard High Salt methods (Sambrook et al. 1989). Detection of parasites was made using PCR reactions with the primers HEMO1 and HEMO2 (Perkins and Keller 2001), targeting part of the $18 \mathrm{~S}$ rRNA gene region, and using the primers HepF300 and HepR900 (Ujvari et al. 2004), targeting another partially overlapping part of the $18 \mathrm{~S}$ rRNA gene region. Conditions of the PCR are detailed in Harris et al. (2011). Briefly, PCR cycling for the HEMO primers consisted of $94^{\circ} \mathrm{C}$ for $30 \mathrm{~s}, 48^{\circ} \mathrm{C}$ for $30 \mathrm{~s}$, $72^{\circ} \mathrm{C}$ for $1 \mathrm{~min}$ ( 35 cycles), whereas for the Hep primers annealing temperature was $60^{\circ} \mathrm{C}$. A total of 133 samples were analysed using PCR, with negative and positive controls run with each reaction. The positive PCR products obtained were purified and sequenced by a commercial sequencing facility (Macrogen Inc., Seoul, Korea). All fragments were sequenced in both directions. Sequences were deposited in GenBank under the accession numbers JX531906 to JX531973.

Samples from $P$. hispanica from Spain and P. lilfordi from Cabrera Island were used in a previous study using a shorter fragment of the Hepatozoon 18S rRNA gene (Harris et al. 2012). In this study we amplified and sequenced the missing region using the Hep primers to be able to compare the full $1401 \mathrm{bp}$ length (see Table 1).

\section{Phylogenetic analysis}

Consensus sequences for each individual were created by combining the sequences of the two partially overlapping 18S rRNA gene regions and analysed using Geneious 5.6.2 (Drummond et al. 2012). Some sequences had heterozygous positions, which could indicate the existence of different Hepatozoon isolates in the same individual, or variation within the multiple copies of the 18S rRNA gene within the same parasite. These were given the corresponding IUPAC code letter.

We conducted two separate analyses, one containing a longer 18S rRNA gene segment (1401 bp, combining sequences obtained with Hep and HEMO primers) (Harris et al. 2011, Maia et al. 2011) and the other containing a shorter 18S rRNA gene segment (562 bp, obtained with Hep primers) in order to compare their phylogenetic resolution and because many previously published sequences were available only for the shorter fragment. Since not all samples worked for both fragments, a total of 49 new parasite sequences were obtained for both fragments, whereas 68 parasite sequences were obtained only for the shorter segment.

Sequences were blasted in GenBank and all matched known Hepatozoon sequences. Hepatozoon sequences available were downloaded from GenBank and those representing different Hepatozoon haplotypes for the major clades were included in the phylogenetic analysis (see Fig. 2). In addition, the newly available sequences from other adeleorinid parasites were also included (HQ224961 Babesiosoma stableri Schmittner et McGhee, 1961, HQ224957 and HQ224958 Dactylosoma ranarum Labbé, 1894, and HQ224959 Haemogregarina balli Paterson et Desser, 1976). Although there is a sequence from Hemolivia mariae Smallridge et Paperna, 1997 available in GenBank (JN211118), it was not included in the analysis because it only 
Table 1. Summary of the samples analysed in this study. ${ }^{1}$

\begin{tabular}{|c|c|c|c|c|c|c|c|c|}
\hline \multirow[b]{2}{*}{ Host } & \multirow[b]{2}{*}{ Country } & \multirow[b]{2}{*}{$\begin{array}{l}\text { Sample size } \\
\text { (males/females) }\end{array}$} & \multicolumn{4}{|c|}{ Molecular screening } & \multicolumn{2}{|c|}{ Microscopic examination } \\
\hline & & & $\begin{array}{l}\text { N positives } \\
\text { (males/females) }\end{array}$ & Prevalence $(\%)$ & HEMO & Hep & $\begin{array}{l}\text { Positives analysed } \\
\text { (males/females) }\end{array}$ & $\begin{array}{l}\text { Mean intensity \% } \\
(\min -\max )\end{array}$ \\
\hline Algyroides marchi & Spain & 66 & 29 & 44 & 19 & 29 & $7(3 / 4)$ & $0.57(0.17-2.05)$ \\
\hline Podarcis bocagei & Portugal & $26(14 / 12)$ & $17(10 / 7)$ & 65 & 13 & 17 & $6(3 / 3)$ & $2.95(0.10-6.88)$ \\
\hline Podarcis bocagei & Spain & $12(7 / 5)$ & $5(2 / 3)$ & 42 & 4 & 5 & $5(2 / 3)$ & $0.49(0.25-1.14)$ \\
\hline Podarcis hispanica type $1 \mathrm{~b} *$ & Spain & $19(10 / 9)$ & $19(10 / 9)$ & 100 & 17 & 19 & $19(10 / 9)$ & $2.52(0.30-11.55)$ \\
\hline \multirow[t]{2}{*}{ Podarcis lilfordi ${ }^{*}$} & Cabrera & $10(3 / 7)$ & $7(3 / 4)$ & 70 & 7 & $2 * *$ & $7(2 / 5)$ & $0.97(0.42-2.77)$ \\
\hline & & 133 & 77 & 58 & 60 & 72 & $44(20 / 24)$ & $1.79(0.10-11.55)$ \\
\hline
\end{tabular}

${ }^{1}$ Prevalence is based on positive PCR results; * samples from Harris et al. (2012); ** as described by Harris et al. (2012); HEP primers preferentially amplified Sarcocystis in double infections.

partially overlapped with the short fragment used here. The final alignments contained 113 Hepatozoon sequences that were 1401 bp long, whereas the second analysis contained 151 Hepatozoon sequences that were 562 bp long. Sequences were aligned with the ClustalW algorithm using default parameters implemented in Geneious 5.6.2 and checked by eye.

Two different phylogenetic analyses (Maximum Likelihood and Bayesian Inference) were conducted. Maximum Likelihood (ML) analysis with random sequence addition (100 replicate heuristic searches) was used to assess evolutionary relationships, using the software PhyML 3.0 (Guindon et al. 2010). Support for nodes was estimated using the bootstrap technique (Felsenstein 1985) with 500 replicates. The AIC criterion conducted in Modeltest 3.06 (Posada and Crandall 1998) was used to choose the model of evolution and the parameters employed (TVM $+\mathrm{G}$ for the shorter fragment, $\mathrm{TVM}+\mathrm{I}+\mathrm{G}$ for the longer fragment).

Bayesian analysis was implemented using Mr. Bayes v.3.1 (Huelsenbeck and Ronquist 2001) with parameters estimated as part of the analysis. The analysis was run for $10 \times 10^{6}$ generations, saving one tree each 1000 generations. The log-likelihood values of the sample point were plotted against the generation time and all the trees prior to reaching stationary were discarded, ensuring that burn-in samples were not retained. Remaining trees were combined in a 50\% majority consensus tree, in which frequency of any particular clade represents the posterior probability (Huelsenbeck and Ronquist 2001). Adelina Hesse, 1911 was used as an outgroup for rooting the phylogenetic tree (Morrison 2009). Pairwise uncorrelated differences (p-distance) were estimated using MEGA 5.05 (Tamura et al. 2011).

In order to facilitate the analysis of the phylogenetic relationships within lineage 2 isolates, a network was made using only the longer sequences from this lineage. The network was produced using a Median-Joining analysis with default parameters in the software Network 4.6.1.0 (Bandelt et al. 1999).

\section{Microscopic examination}

Blood smears were examined using an Olympus CX41 microscope with an in-built digital camera (SC30). Several photomicrographs per slide were taken at $400 \times$ magnification and stitched using cell ${ }^{\wedge} \mathrm{B}$ software (basic image-acquisition and archiving software, Olympus, Germany). Intensity of infection, i.e. the percentage of infected cells within a host, was estimated as percentage based on counts of haemogregarines per 2000 cells for a total of 44 blood smears (Table 1). Counts were done using the manual cell counter plug-in available in the image processing software ImageJ ver. 1.44p (Abramoff et al. 2004). Fig. 1 shows intracellular gamonts of Hepatozoon infecting the four lizard species.

\section{Statistics}

Comparison in prevalence between sexes per locality was assessed using Fisher's Exact Test, which is more appropriate than a chi-square test in cases of $2 \times 2$ tables with low frequencies (Zar 2009). Correlation between host body size (snout-vent length) and parasitemia levels was investigated using a Spearman correlation. All analyses were performed using R (R Development Core Team 2009).

\section{RESULTS}

Of the 133 samples analysed using molecular markers, 77 were positive, which gives an overall prevalence of $58 \%$. Primer performance differed between the two sets used in this study. Overall, the Hep primers performed better at detecting, amplifying and sequencing Hepatozoon than the HEMO primers, except in double infection cases (see Table 1). Prevalence of Hepatozoon sp. was highest in Podarcis hispanica (100\%), followed by P. lilfordi (70\%), P. bocagei Seoane from Portugal (65\%) and Algyroides marchi Valverde (44\%) with P. bocagei from Spain having the lowest prevalence (42\%) (Table 1). There was no significant variation of prevalence between sexes (Fisher's Exact Test, in all cases $\mathrm{p}>0.05$ ). Intensity of infection, i.e. the percentage of infected cells, varied across and within host species and geographical locations. Algyroides marchi and P. bocagei from Spain showed the lowest haemogregarine intensities, whereas the highest intensities were found in P. bocagei from Portugal and $P$. hispanica type $1 \mathrm{~b}$. There was no relation between snout-vent length and intensity of infection in any of the populations analysed (Spearman Correlation, in all cases $\mathrm{p}>0.5)$.

The two phylogenetic methodologies used to estimate phylogeny produced similar tree topologies and thus only one is presented (Fig. 2). Two main lineages were found, one composed of a single Hepatozoon isolate from the lacertid P. bocagei from Portugal (lineage 1) and other lineage of the remaining Iberian and Cabrera Hepatozoon isolates (lineage 2) (see Fig. 2). Lineage 1 is closely related to one of the lineages found in North Africa that also infects a lacertid host (Timon tangitanus Boulenger). In contrast, all other Hepatozoon isolates (from A. marchi, $P$. hispanica, P. bocagei and P. lilfordi) are closely related to a generally unresolved Hepatozoon lineage found 


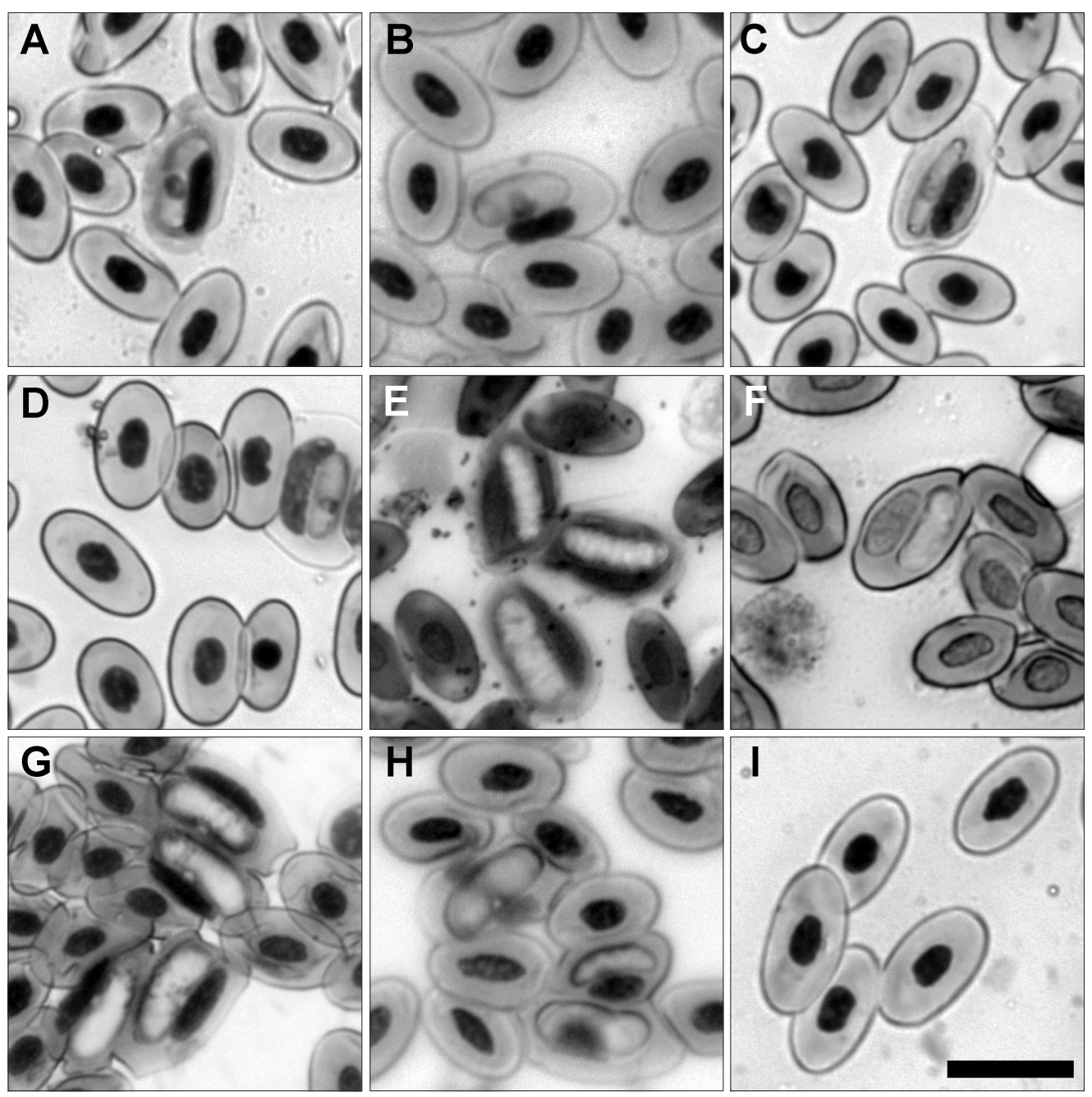

Fig. 1. Hepatozoon parasites infecting erythrocytes from Algyroides marchi from Spain (DB9365AmSP and DB9379AmSP) (A, B), Podarcis lilfordi from Cabrera Island (DB10319P1CA and DB10553P1CA) (C, D), P. bocagei from Portugal (3251PbPO and 3284PbPO) (E, F), and P. hispanica from Spain (5204PhSP, 5212PhSP) (G, H). Normal, uninfected, lizard erythrocytes (from DB9171AmSP) (I). Scale bar $=10 \mu \mathrm{m}$.

in skinks (Eumeces algeriensis Peters and Chalcides polylepis Boulenger) and several lacertids from North Africa (including another Podarcis species, P. vaucheri Boulenger), and lacertids from the Iberian Peninsula and Balearic Islands (Fig. 2).

Sequence divergence between the isolate found in the lineages 1 and 2 is $1.3-1.6 \%$ (for the longer segment) and $3.1-3.3 \%$ (for the shorter segment). Within the lineage 2, all $A$. marchi isolates form a monophyletic subgroup, whereas relationships between isolates from $P$. bocagei and $P$. hispanica are not well resolved. The network of the unresolved lineage 2 shows that Hepatozoon parasites from $P$. bocagei and $P$. hispanica can be found in two groups, whereas those from $A$. marchi form a unique subgroup (Fig. 3).

\section{DISCUSSION}

Our results show that both parasite prevalence and intensity vary between and within host species and their geographical locations. Overall, prevalence was high in our study, which is in accordance with the morphological studies on lizards from the same region. A microscopic survey of Podarcis carbonelli Pérez Mellado and P. bocagei from northwest Portugal showed similar prevalence of haemogregarines, $69.7 \%$ and $74.7 \%$, respectively, although intensities were not reported (Roca and Galdón 2010). Another microscopic survey on Podarcis muralis Laurenti from Spain found $58.1 \%$ prevalence and intensity levels of less than $0.5 \%$ (Amo et al. 2005a).

In this study we found varying prevalence values for parasites infecting the genus Podarcis Wagler, with all individuals infected from one population of $P$. hispanica, whereas for $P$. bocagei from Spain less than half of the individuals analysed were infected. In fact, within $P$. bocagei from Portugal we found high prevalence $(65 \%$, $\mathrm{n}=26)$ and high mean intensity levels $(2.95 \%, \mathrm{n}=6)$, whereas in Spain prevalence and intensity values were much lower $(42 \%, \mathrm{n}=12$, and $0.49 \%, \mathrm{n}=5$, respectively). 


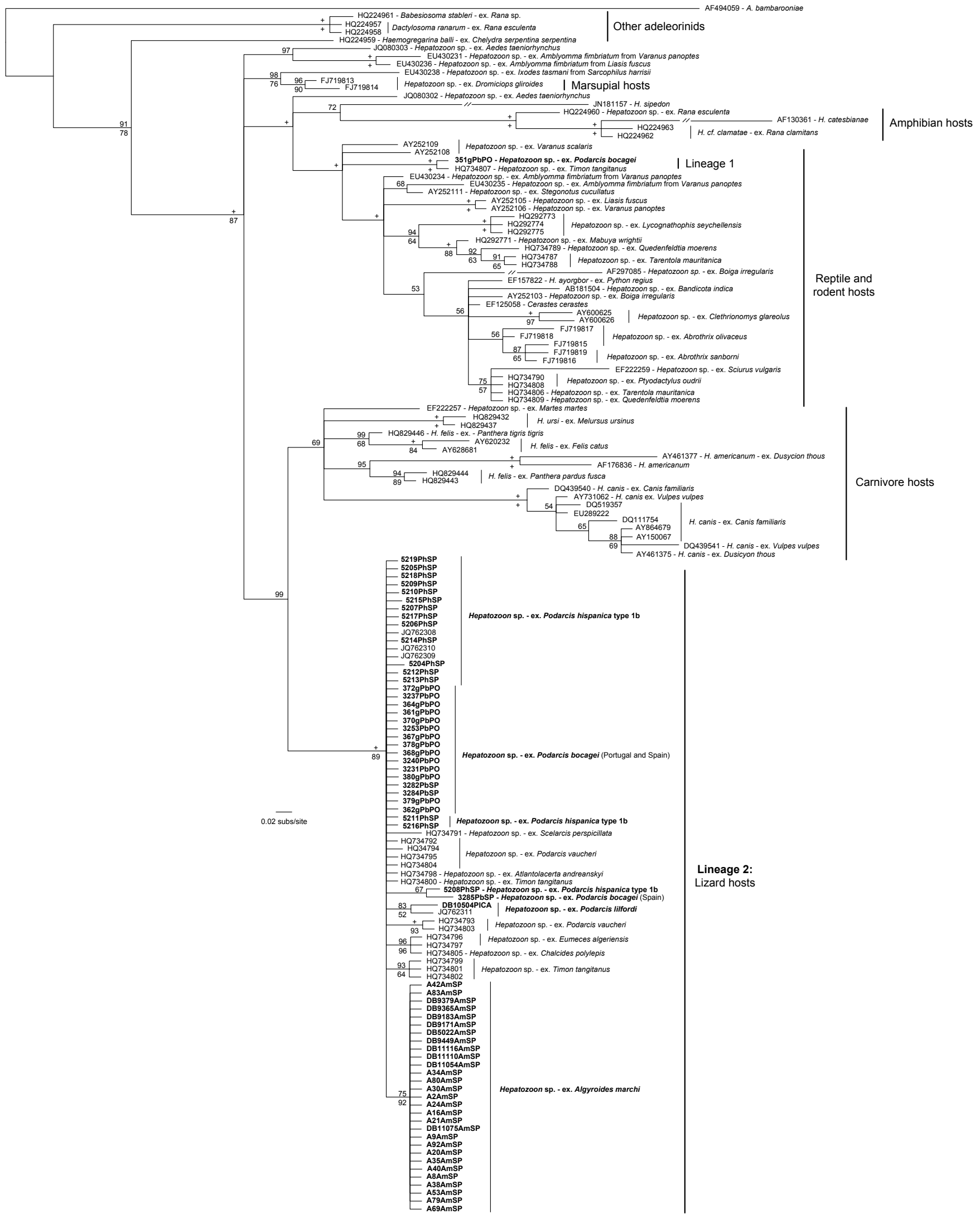

Fig. 2. Bayesian estimate of relationships of Hepatozoon species based on 562 bp $18 \mathrm{~S}$ rRNA gene sequences. Bayesian posterior probabilities are given above the nodes and ML bootstrap values below them. When both values were $100 \%$, this is indicated with $\mathrm{a}+$. New haplotypes from this study are in bold. The branches of JN181157, AF130361 and AF297085 were shortened by $50 \%$. 


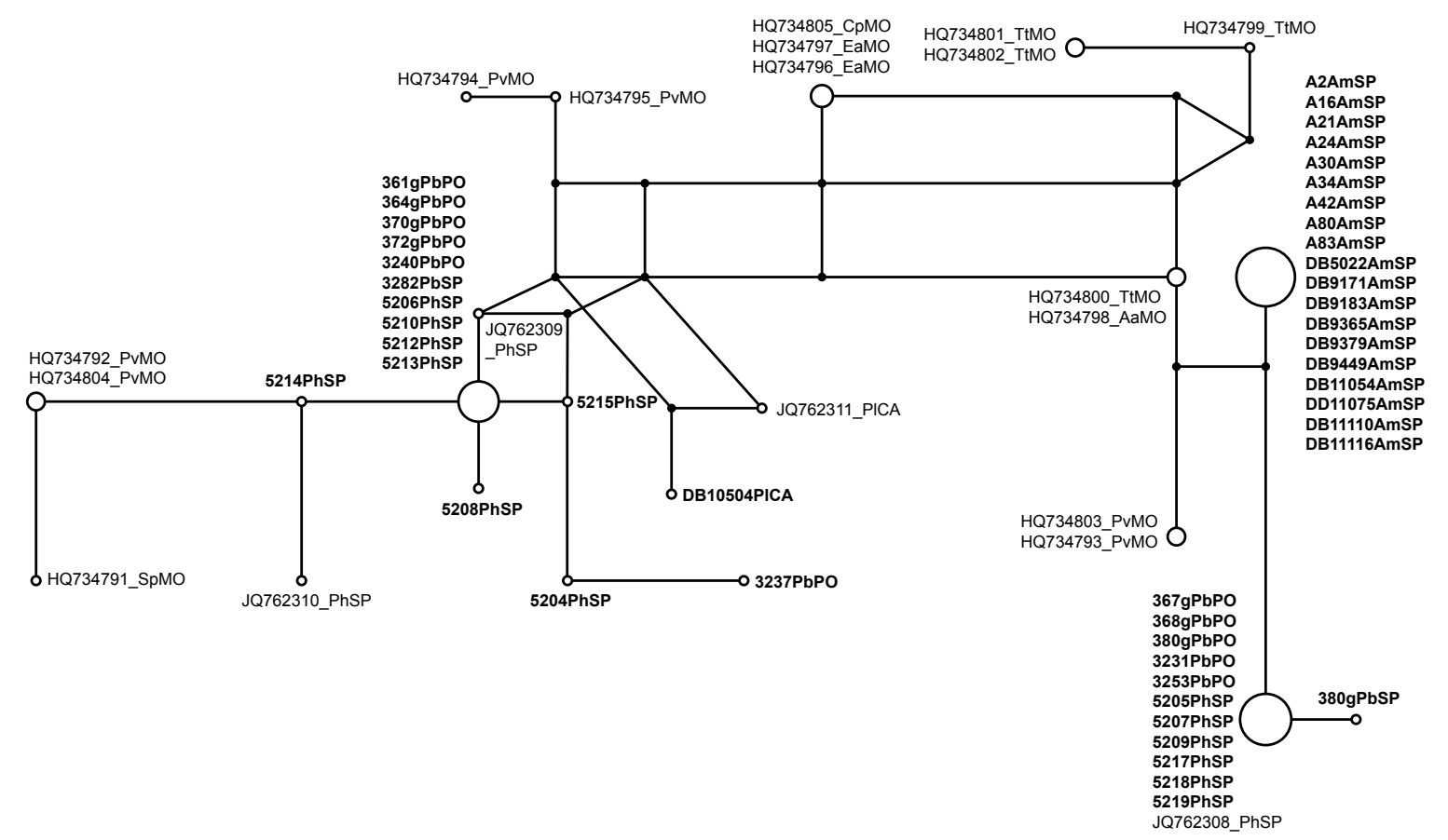

Fig. 3. Median-Joining Network analysis of lineage 2, using 1401 bp 18S rRNA gene sequences. New haplotypes from this study are in bold. Last four letters indicate host species initials (first two letters) and country (last two letters).

Nevertheless, since only 12 individuals were screened for P. bocagei in Spain, this difference could be biased by non-representative sampling.

Interestingly, mean intensity values for $P$. bocagei are similar to those of $P$. muralis reported by Amo et al. (2005a). Differences in prevalence and intensity of haemoparasites (either between or within host species) could be associated with factors such as microhabitat characteristics (Davis et al. 2012), feeding habits and seasonality. Indeed, seasonality has been shown to be associated with differences in parasite load (Salkeld and Schwarzkopf 2005, Santos et al. 2005). However, since our samples were collected during different seasons and in different years, we cannot draw conclusions regarding this. This could be investigated by conducting large-scale sampling of individuals from several species from a single location and from the same species from different locations at different seasons. Also, we still know little about the influence that these parasites may have on host health and fitness, and this could also be investigated using immunological and performance tests (Sacchi et al. 2007).

To our knowledge, this is the first assessment of the occurrence of haemogregarines in the species Algyroides marchi. There is only a record of haemogregarine infection in Algyroides nigropunctatus Duméril et Bibron (from Italy), with a mean intensity of about $0.2 \%$ (no prevalence records are provided), in a survey performed with the purpose of comparing counts and morphology of leukocytes in 12 lizards species (Sacchi et al. 2011). In our study, intensity levels in $A$. marchi were very similar to those found in its Italian relative (mean $=0.57$, min.max.: 0.17-2.05).
Our study highlights the utility of the short 18S rRNA fragment amplified using the Hep set of primers for largescale molecular surveys of Hepatozoon parasites, since the estimate of phylogenetic relationships is similar to those retrieved using the longer fragment. In addition, Hep primers usually perform better at detecting infections and normally yield better quality sequences. Although a larger fragment provides more genetic information and acts as a safety net against contamination, because it requires two separate PCRs, the sole use of Hep primers provides similar estimates of phylogenetic relationships at lower cost. Nonetheless, as realized recently, Hep primers have the potential of amplifying DNA of other apicomplexan parasites and in double infection some primers may preferentially amplify other apicomplexans, such as Eimeria Schneider, 1875 and Sarcocystis Lankester, 1882 coccidia (Harris et al. 2012). Hence, in these situations samples should also be tested with the HEMO primers to check for the presence of haemogregarines, since these are more specific primers.

The phylogenetic relationships estimated for Hepatozoon parasites remain similar to those previously described (Maia et al. 2011, Harris et al. 2012), despite the addition of new sequences for the 18S rRNA gene in this study. However, the present phylogenetic analysis increases the known geographical distribution of this group and the number of host species infected by these parasites. One lineage from North Africa, previously known only from the lacertid host Timon tangitanus, appears to be related with one of the new isolates from P. bocagei, whereas all the other new isolates fall in a group with another lineage from North Africa found in lacertids and skinks. 
The occurrence of two very distinct genetic lineages of Hepatozoon isolates from the same host species demonstrates that a single host species can harbour genetically distinct isolates. Since the 18S rRNA is a slow evolving gene, the high sequence divergence found between the lineages 1 and 2 could indicate that these represent different, unrelated species of Hepatozoon. Unfortunately, no blood smear was made at the time of collection for the unique sample from lineage 1 from $P$. bocagei and thus it is not possible to couple microscopy with phylogeny for this lineage.

Still, this result emphasizes that Hepatozoon may have low host-specificity (Telford et al. 2001), at least concerning some intermediate host species such as $P$. bocagei. Thus, the transmission cycle should be fully studied in the future, by collecting from a single location (with known parasitic infections) both the vertebrate host and the potential vectors that are the definitive hosts (arthropods which are often found attached to the intermediate host, such as ticks and mites, but also mosquitoes and reduviid bugs - Telford 2009). This is especially important since relatively high degree of host-parasite association of various species of Apicomplexa with their definitive hosts have been observed recently (Barta et al. 2012).

In contrast, the fact that Hepatozoon isolates from Algyroides form a monophyletic subgroup within the lineage 2 demonstrates some degree of strict intermediate host specificity. Finally, the phylogenetic relationships between the isolates found in P. bocagei and P. hispanica remain largely unresolved and given that the hosts are closely related species (together forming a species complex - see Pinho et al. 2008) it is possible that these hosts share the same parasite communities. Nonetheless, further research should be conducted using faster evolving genes, to obtain a better resolution of this lineage.

By including other adeleorinid sequences in our phylogeny, we demonstrate in accordance with Barta et al. (2012) that these are clearly distinct from our Hepatozoon sequences, thus discarding the possibility of the lineage 1 being from other adeleorinids, a possibility that could not previously be rejected (Maia et al. 2011). Support levels for the major groups within Hepatozoon are also low, further indicating that more data are necessary prior to any taxonomic changes being appropriate.

Finally, our phylogeny also presents some incongruences in sequences deposited in GenBank. For example, the sequence identified as the snake Cerastes cerastes Linnaeus in GenBank (EF125058) is actually a Hepatozoon sp., presumably accidentally amplified instead of the host. This kind of erroneous amplification of parasites instead of hosts, and subsequent mislabelling in databases, has also been recorded for flatworms (Heneberg 2012), and further highlights the need for care when comparisons are made with sequences from GenBank.

Acknowledgements. JPMCM is supported by a Fundação para a Ciência e a Tecnologia (FCT) $\mathrm{PhD}$ grant (SFRH/ $\mathrm{BD} / 74305 / 2010$ ) co-financed by FSE and POPH and EU, and AP by a FCT postdoctoral fellowship (SFRH/BPD/26546/2006). Thanks are due to our colleagues from CIBIO who helped with the fieldwork, and to the people and institutions that made possible to obtain samples from Portugal, Spain and Cabrera Island. Especially, we thank A. Kaliontzopoulou, M.A. Carretero and E. García-Muñoz for collecting samples.

\section{REFERENCES}

Abramoff M.D., Magalhães P.J., Ram S.J. 2004: Image processing with ImageJ. Biophotonics Int. 11: 36-42.

Álvarez-Calvo J.A. 1975: Nuevas especies de hemococcidios en lacértidos españoles. Cuadernos de Cienc. Biol. 4: 207-222.

Amo L., Fargallo J.A., Martínez-Padilla J., Millán J., López P., Martín J. 2005b: Prevalence and intensity of blood and intestinal parasites in a field population of a Mediterranean lizard, Lacerta lepida. Parasitol. Res. 96: 413-417.

Amo L., López P., Martín J. 2004: Prevalence and intensity of haemogregarinid blood parasites in a population of the Iberian rock lizard, Lacerta monticola. Parasitol. Res. 94: 290-293.

Amo L., López P., Martín J. 2005a: Prevalence and intensity of haemogregarine blood parasites and their mite vectors in the common wall lizard, Podarcis muralis. Parasitol. Res. 96: 378-381.

Bandelt H.J., Forster P., Röhl A. 1999: Median-joining networks for inferring intraspecific phylogenies. Mol. Biol. Evol. 16: 37-48.

Baneth G., Mathew J.S., Shkap V., Macintire D.K., Barta J.R., EwIng S.A. 2003: Canine hepatozoonosis: two disease syndromes caused by separate Hepatozoon spp. Trends Parasitol. 19: 27-31.

Barta J.R., Ogedenbre J.D., Martin D.S., Smith T.G. 2012: Phylogenetic position of the adeleorinid coccidia (Myzozoa, Apicomplexa, Coccidia, Eucoccidiorida, Adeleorina) inferred using 18S rDNA sequences. J. Eukaryot. Microbiol. 59: 171-180.
Criado-Fornelio A., Buling A., Casado N., Gimenez C., Ruas J., Wendt L., da Rosa-Freitas N., Pinheiro M., Rey-Valeiron C., Barba-Carretero J.C. 2009: Molecular characterization of arthropod-borne hematozoans in wild mammals from Brazil, Venezuela and Spain. Acta Parasitol. 54: 187-193.

Criado-Fornelio A., Martinez-Marcos A., Buling-Saraña A., Barba-Carretero J.C. 2003: Molecular studies on Babesia, Theileria and Hepatozoon in southern Europe: Part II. Phylogenetic analysis and evolutionary history. Vet. Parasitol. 114: 173194.

Criado-Fornelio A., Rey-Valeiron C., Buling A., Barba-CarRetero J.C., Jefferies R., Irwin P. 2007: New advances in molecular epizootiology of canine hematic protozoa from Venezuela, Thailand and Spain. Vet. Parasitol. 144: 261-269.

Criado-Fornelio A., Ruas J.L., Casado N., Farias N., Soares P., Muller G., Brum J.G.W., Berne M.E.A., Buling A., BarbaCarretero J.C. 2006: New molecular data on mammalian Hepatozoon species (Apicomplexa: Adeleorina) from Brazil and Spain. J. Parasitol. 92: 93-99.

Davis J.R., Boyle S.A., Khan A.A., Gay A.L.J., Grisham J.M., LUQUE L.E. 2012: Snake parasitism in an urban old-growth forest. Urban Ecosyst. 15: 739-752.

Drummond A.J., Ashton B., Buxton S., Cheung M., Cooper A., Duran C., Field M., Heled J., Kearse M., Markowitz S., 
Moir R., Stones-Havas S., Sturrock S., Thierer T., Wilson A. 2012: Geneious v5.6. Available from http://www.geneious.com

Felsenstein J. 1985: Confidence and phylogenies: an approach using the bootstrap. Evolution 39: 783-791.

Garcia P., Acedo M.C., Lopez J.J., Sanchis M.C., Morillas F. 1990: Identification of Hepatozoon canis (James, 1905) in Spain. Epidemiological study of an enzootic in La Carolina, Jaen. Investigación Agraria, Producción y Sanidad Animales 5: 75-89.

Guindon S., Dufayard J.F., Lefort V., Anisimova M., Hordijk W., Gascuel O. 2010: New algorithms and methods to estimate maximum-likelihood phylogenies: assessing the performance of PhyML 3.0. Syst. Biol. 59: 307-321.

Harris D.J., Maia J.P.M.C., Perera A. 2011: Molecular characterization of Hepatozoon species in reptiles from the Seychelles. J. Parasitol. 97: 106-110.

Harris D.J., Maia J.P.M.C., Perera A. 2012: Molecular survey of Apicomplexa in Podarcis wall lizards detects Hepatozoon, Sarcocystis and Eimeria species. J. Parasitol. 98: 592-597.

Heneberg P. 2012: On the robustness of phylogenetic analyses: can flatworm 18S rDNA hide between $18 \mathrm{~S}$ rDNAs of a single mollusc genus? Mol. Phyl. Evol. In Press. DOI: 10.1016/j.ympev.2012.08.003

Huelsenbeck J.P., Ronquist F. 2001: MrBayes: Bayesian inference of phylogeny. Bioinformatics 17: 754-755.

Jovani R., Amo L., Arriero E., Krone O., Marzal A., Shurulinkov P., Tomás G., Sol D., Hagen J., López P., Martín J., Navarro C., Torres J. 2004: Double gametocyte infections in apicomplexan parasites of birds and reptiles. Parasitol. Res. 94: 155-157.

Lledó L., Giménez-Pardo C., Domínguez-Peñafiel G., Sousa R., Gegúndez M.I., Casado N., Criado A. 2010: Molecular detection of Hemoprotozoa and Rickettsia species in arthropods collected from wild animals in the Burgos province, Spain. VectorBorne Zoon. Dis. 10: 735-738.

Maia J.P.M.C., Harris D.J., Perera A. 2011: Molecular survey of Hepatozoon species in lizards from North Africa. J. Parasitol. 97: 513-517.

Martín J., Civantos E., Amo L., López P. 2007: Chemical ornaments of male lizards Psammodromus algirus may reveal their parasite load and health state to females. Behav. Ecol. Sociobiol. 62: $173-179$

Mathew J.S., Van Den Bussche R.A., Ewing S.A., Malayer J.R., Latha B.R., Panciera R.J. 2000: Phylogenetic relationships of Hepatozoon (Apicomplexa: Adeleorina) based on molecular, morphologic, and life-cycle characters. J. Parasitol. 86: 366-372.

Moço T.C., Silva R.J., Madeira N.G., Paduan K.S., Rubini A.S., Leal D.D.M., O’Dwyer L.H. 2012: Morphological, morphometric, and molecular characterization of Hepatozoon spp. (Apicomplexa, Hepatozoidae) from naturally infected Caudisona durissa terrifica (Serpentes, Viperidae). Parasitol. Res. 110: 1393-1401.

Morrison D.A. 2009: Evolution of the Apicomplexa: where are we now? Trends Parasitol. 25: 375-382.

Ortuno A., Castella J., Criado-Fornelio A., Buling A., BarbaCarretero J.C. 2008: Molecular detection of a Hepatozoon species in stray cats from a feline colony in North-eastern Spain. Vet. J. 177: 134-135.

Paterson S., Piertney S.B. 2011: Frontiers in host-parasite ecology and evolution. Mol. Ecol. 20: 869-871.

Perkins S.L., Keller A.K. 2001: Phylogeny of nuclear small subunit rRNA genes of hemogregarines amplified with specific primers. J. Parasitol. 87: 870-876.

Pinho C., Harris D.J., Ferrand N. 2008: Non-equilibrium estimates of gene flow inferred from nuclear genealogies suggest that Iberian and North African wall lizards (Podarcis spp.) are an assemblage of incipient species. BMC Evol. Biol. 8: 63.
Posada D., Crandall K.A. 1998: Modeltest: testing the model of DNA substitution. Bioinformatics 14: 817-818.

Poulin R., Mouillot D. 2005: Combining phylogenetic and ecological information into a new index of host specificity. J. Parasitol. 91: 511-514.

R Development Core Team 2009: R: a language and environment for statistical computing. R Foundation for Statistical Computing, Vienna, Austria. Available at: http://www.R-project.org.

Roca V., Galdón M.A. 2010: Haemogregarine blood parasites in the lizards Podarcis bocagei (Seoane) and P. carbonelli (PérezMellado) (Sauria: Lacertidae) from NW Portugal. Syst. Parasitol. 75: 75-79.

Sacchi R., Rubolini D., Gentilli A., Pupin F., Razzetti E., Scal S., Galeotti P., Fasola M. 2007: Morph-specific immunity in male Podarcis muralis. Amphibia-Reptilia 28: 408-412.

Sacchi R., Scali S., Cavirani V., Pupin F., Pellitteri-Rosa D., Zuffi M.A.L. 2011: Leukocyte differential counts and morphology from twelve European lizards. Ital. J. Zool. 78: 418-426.

Salkeld D.J., Schwarzkopf L. 2005: Epizootiology of blood parasites in an Australian lizard: a mark-recapture study of a natural population. Int. J. Parasitol. 35: 11-18.

Sambrook J., Fritsch E.F., Maniatis T. 1989: Molecular Cloning: A Laboratory Manual. Cold Spring Harbour Press, New York, 545 pp.

Santos M.M. de V., O’Dwyer L.H., da Silva R.J. 2005: Seasonal variation of Hepatozoon spp. (Apicomplexa, Hepatozoidae) parasitemia from Boa constrictor amarali (Serpentes, Boidae) and Hydrodynastes gigas (Serpentes, Colubridae). Parasitol. Res. 97: 94-97.

Sмith T.G. 1996: The genus Hepatozoon (Apicomplexa: Adeleina) J. Parasitol. 82: 565-585.

Smith T.G., Desser S.S. 1997: Phylogenetic analysis of the genus Hepatozoon Millet 1908 (Apicomplexa: Adeleorina). Syst. Parasitol. 36: 213-221.

Tabar M., Altet L., Francino O., Sánchez A., Ferrer L., Roura X. 2008: Vector-borne infections in cats: molecular study in Barcelona area (Spain). Vet. Parasitol. 151: 332-336.

Tamura K., Peterson D., Peterson N., Stecher G., Nei M., KuMAR S. 2011: MEGA5: Molecular Evolutionary Genetics Analysis using Maximum Likelihood, Evolutionary Distance, and Maximum Parsimony methods. Mol. Biol. Evol. 28: 2731-2739.

Telford S.R. 2009: Hemoparasites of the Reptilia: Color Atlas and Text. CRC Press, Taylor and Francis Group, Boca Raton, Florida, 394 pp.

Telford S.R., Wozniak E.J., Butler J.F. 2001: Haemogregarine specificity in two communities of Florida snakes, with descriptions of six new species of Hepatozoon (Apicomplexa: Hepatozoidae) and possible species of Haemogregarina (Apicomplexa: Haemogregarinidae). J. Parasitol. 87: 890-905.

Tomé B., Maia J.P.M.C., Harris D.J. 2012: Hepatozoon infection prevalence in four snake genera: influence of diet, prey parasitaemia levels or parasite type? J. Parasitol. 98: 913-917.

Ujvari B., Madsen T., Olsson M. 2004: High prevalence of Hepatozoon spp. (Apicomplexa, Hepatozoidae) infection in water pythons (Liasis fuscus) from tropical Australia. J. Parasitol. 90: 670-672.

Yabsley M.J., McKibben J., Macpherson C.N., Cattan P.F., Cherry N.A., Hegarty B.C., Breitschwerdt E.B., O’Connor T., Chandrashekar R., Paterson T., Perea M.L., Ball G., Friesen S., Goedde J., Henderson B., Sylvester W. 2008: Prevalence of Ehrlichia canis, Anaplasma platys, Babesia canis vogeli, Hepatozoon canis, Bartonella vinsonii berkhoffii, and Rickettsia spp. in dogs from Grenada. Vet. Parasitol. 151: 279-285.

Zar J.H. 2009: Biostatistical Analysis. Fourth Edition, Prentice Hall, Upper Saddle River, New Jersey, 663 pp. 